\title{
論文
}

$$
\begin{gathered}
\text { 다비행체 편대비행을 위한 유도법칙 및 시뮬레이션에 관한 연구 } \\
\text { 노태수*, 전경언** }
\end{gathered}
$$

\section{A Study on Guidance Law Design and Simulation of Multiple UAV Formation Flying}

Tae Soo No* and Gyeong Eon Jeon**

\begin{abstract}
A guidance scheme for controlling the relative geometry of multiple flight vehicle formation flying is proposed. Each flight vehicle in the formation takes the roles of leader and follower simultaneously except for the formation leader. In this scheme, the flight commands for a leader are shared by all the followers and this leaders to a synchronized flight of all flight vehicles comprising the formation. Lyapunov stability theorem is used to obtain the guidance law. High fidelity nonlinear simulation results are presented to show the effectiveness of the proposed guidance law using a reconnaissance and surveillance mission example.

\section{초 록}

본 논문에서는 다수 비행체의 편대 비행 시 상호 기하학적 관계 유지에 필요한 유도 법 칙과 비선형 시뮬레이션 결과를 제시하였다. 편대 내의 각 비행체는 편대 Leader를 제외하 고 모두 Leader와 Follower의 역할을 동시에 맡으며, Leader에 의한 명령은 모든 Follower 에게 분배되고 따라서 편대를 이루는 모든 비행체들의 동시기동비행 Synchronized Flight) 을 가능하게 한다. 편대 비행 유도 법칙은 가까운 미래 시각에 예상되는 기하학적 오차 그리고 Lyapunov 안정성 이론에 근거하여 도출하였고, 정찰과 감시 임무 예제에 관한 고 정밀 비선형 시뮬레이션 결과를 통하여 제안된 유도 법칙의 성능을 검증하였다.
\end{abstract}

Key Words : Guidance Law(유도법칙), Multi-Vehicle Formation Flight(다비행체 편대 비행), Cascade Type(순차구조), Lyapunov Stability(리아푸노프 안정성)

\section{I. 서 론}

다수의 무인기로 이루어진 편대 비행은 정찰 과 감시, 목표물 탐지, 전투 피해 평가와 같은 군

†2008년 4월 21일 접수 2008년 7월 17일 심사완료

* 정회원, 전북대학교 항공우주공학과

교신저자, E-mail : rotthee@chonbuk.ac.kr

전북 전주시 덕진구 덕진동 1 가 664-14

** 정회원, 전북대학교 항공우주공학과 대학원
사적 임무뿐 만 아니라 구조, 원격 탐사, 통신 중 계 서비스 등과 같은 민간 임무에 적용이 가능하 고[1-3], 최근 일부 성공적인 비행 시험 결과도 보고되고 있는 실정이다[4-7]. 그러나 편대 비행 시스템의 완전 자동화를 위해서는 각 개별 비행 체의 제어, 편대 수준의 제어, 모델링 및 시뮬레 이션, 편대 비행을 위한 유도법칙 등 여러 가지 분야에서 심도 있는 연구 노력들이 요구되고 있 다[8 11]. 예를 들어, 충돌회피와 편대 재배치 등 
을 자율적으로 수행할 필요가 있으며, 이러한 기 능들은 편대 내에서의 통신망(Communication) 구조, 탑재 컴퓨터의 성능, 각 비행체에 탑재되는 센서의 종류에 매우 의존적이다. 또한 단일 비행 체뿐만 아니라 편대 내에서 작동자의 명령들과 비행체의 시스템사이의 응답을 실시간 체크할 수 있는 기술적인 문제들도 요구된다[14]. 편대 비행 제어 및 유도 관점에서 각각의 비행체는 외부로 부터 명령을 받고 수행할 수 있는 기능이 필요하 다. 따라서 전체적인 편대 비행 제어 시스템의 복잡도와 강건성은 통신망의 구성 및 탑재 컴퓨 터의 계산 성능에 따라 결정된다.

본 연구에 있어서 편대 비행은 일정한 기하학 적 형상을 유지하고 있는 비행체들 사이의 거리 및 위치가 일정하게 유지되도록 제어되면서 비행 하는 것을 의미한다. 이러한 편대 비행에 필요한 여러 제어 기법들에 관한 연구가 이루어져 있다 [15-19]. 대부분의 편대 비행 제어 법칙들은 Leader-Follower 구조에 근거를 두고 설계되나 참고문헌 [15]의 경우 Leader 비행체의 고장에 강건한 소위 Leaderless 편대 비행 기법을 제안 하였다. Leader-Follower 편대 비행의 장점은 Leader의 비행 궤적을 Follower 비행체가 단순 추종하는 일반적인 Tracking 문제로 간략화 할 수 있다는 점이다. 미사일 유도 법칙도 편대 비 행 제어에 응용되고 있다. 즉, Leader는 피요격 (Target) 물체로 간주하고 Follower는 요격 미사 일로 간주하되 Hit Miss Distance를 일정하게 유 지한다.

본 논문에서는 편대를 유지하기 위한 유도 법 칙의 하나로 Cascade 형태의 제어법칙을 제안하 고자 한다. Follower가 Leader와의 상대위치 및 속도 오차만을 고려하여 뒤에서 추적하는 전통적 인 Leader-Follower 기법과 달리 Follower가 Leader의 비행 정보를 이용하여 유도 명령을 계 산한다. 전체 편대를 다수의 Branch로 구성하고 각각의 Branch는 각각의 비행체가 직렬로 연결 되어 있다고 가정하였다. 또한 각 Branch에 속하 는 개별 비행체는 Leader 및 Follower의 역할을 동시에 수행한다. 물론 편대 전체의 Leader 및 Branch의 최하위 Follower 비행체는 본연의 역 할만 수행한다. 이러한 형태의 편대 비행 방법은 각 개별 비행체가 편대 전체 Leader는 물론 상 위 Leader 비행체 전부의 비행 정보를 공유함으 로서 궁극적으로 편대 전체의 동시 기동 비행 (Synchronized Flight Maneuver)이 가능하다.

\section{ㅍ. 유도 법칙 설계}

\section{1 편대 기하}

본 논문에서는 그림 1 에 보인 형태의 편대 기 하를 고려하고자 한다. 편대 전체는 일정한 기하 학적 형상을 유지하고, 편대 내의 각 비행체는 상호 상대적인 기하를 동시에 유지해야 한다. 단, 편대 전체의 Leader는 임의 비행을 한다고 가정 하였다. UAV $j$ 는 Follower UAV $k$ 의 Leader이 고 두 무인기들은 편대 내에서 $\mathrm{i}$ 번째 가지 (Branch)에 속한다. 본 논문에서의 편대 비행 유 도 법칙은 Follower UAV k에 대한 유도 비행 명령을 찾는 것을 의미한다. Leader UAV j에 필 요한 비행 명령은 UAV j의 Leader UAV 정보를 이용하여 계산이 되었다고 가정한다.

\section{1 유도법칙 설계}

그림 2를 참조하여, Follower UAV k가 Leader $\mathrm{UAV} \mathrm{j}$ 에 대해 $\Delta d^{*}$ 만큼의 상대위치를 유지해야 한다고 가정한다. 만약 $d^{i}$ 와 $v^{i}$ 가 $\mathrm{UAV} \mathrm{i}$ 의 현재 위치와 속도 벡터를 의미하고, 두 $U A V j$ 와 $k$ 가
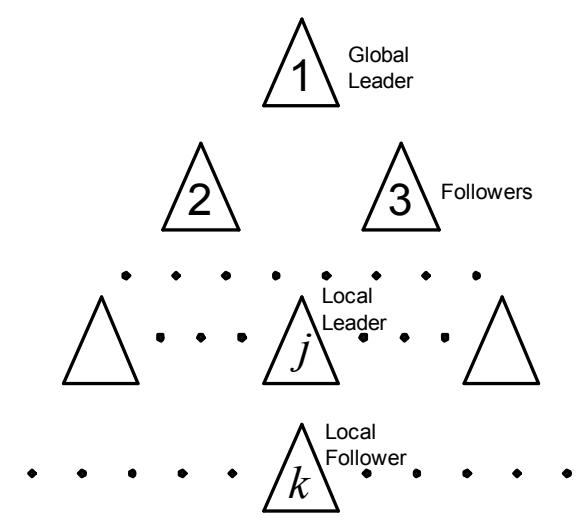

그림 1. Formation Geometry

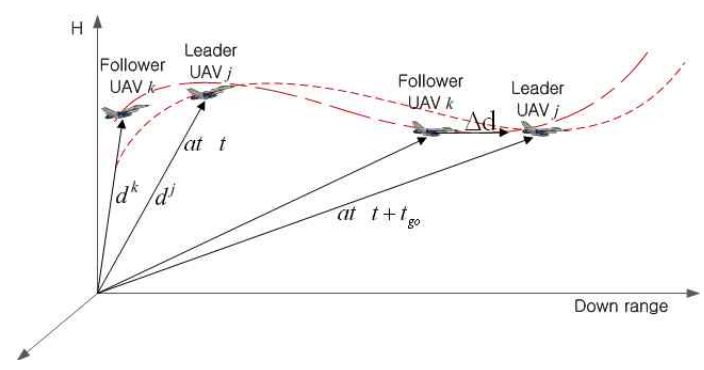

그림 2. Leader-Follower Geometry 
그들 각각의 현재 비행 방향과 속도를 유지한다 면 미래시각 $t_{f}=t+t_{g o}$ 에서 두 비행체간의 상대 위치 벡터는 다음과 같이 나타낼 수 있다.

$$
\Delta d=\left(d^{j}-d^{k}\right)+\left(v^{j}-v^{k}\right) t_{g o}
$$

그리고 다음과 같이 양한정 함수 (Positive Definite Function)를 정의한다.

$$
V=\frac{1}{2}\left(\Delta d-\Delta d^{*}\right) \cdot\left(\Delta d-\Delta d^{*}\right)
$$

만약 Follower UAV k가 식(2)의 값이 매우 작도 록 제어된다면 이는 현재의 상대위치 벡터 $\Delta d$ 가 편대 비행을 위하여 요구되는 상대위치 벡터 $\Delta d^{*}$ 와 거의 같을 것이며 따라서 이것은 편대 기 하가 유지되고 있음을 의미한다.

고정좌표계의 단위벡터 $\left(\boldsymbol{e}_{x}, \boldsymbol{e}_{y}, \boldsymbol{e}_{z}\right)$ 를 사용하여 $\mathrm{UAV} \mathrm{j}$ 및 $\mathrm{k}$ 사이의 편대 기하 오차, UAV j의 절대 속도 및 가속도 벡터를 다음과 같이 정의할 수 있다.

$$
\begin{gathered}
\Delta d-\Delta d^{*}=M_{x} e_{x}+M_{y} e_{y}+M_{z} e_{z} \\
v=v_{x} e_{x}+v_{y} e_{y}+v_{z} e_{z} \\
=(v \cos \gamma \cos \psi) e_{x}+(v \cos \gamma \sin \psi) e_{y}-(v \sin \gamma) e_{z} \\
\frac{d v}{d t}=(\dot{v} \cos \gamma \cos \psi-\dot{v} \gamma \sin \gamma \cos \psi-v \dot{\psi} \cos \gamma \sin \psi) e_{x} \\
+(\dot{v} \cos \gamma \sin \psi-v \dot{\gamma} \sin \gamma \sin \psi+v \dot{\psi} \cos \gamma \cos \psi) e_{y} \\
-(\dot{v} \sin \gamma+v \dot{v} \cos \gamma) \boldsymbol{e}_{z}
\end{gathered}
$$

$\mathrm{UAV}$ 에 고정된 제어축 좌표계 (Control axis frame)의 단위 벡터 $\left(\boldsymbol{e}_{v}, \boldsymbol{e}_{\psi}, \boldsymbol{e}_{\gamma}\right)$ 를 다음과 같이 정 의한다. $e_{v}$ 는 현재 속도벡터 방향으로의 단위 방 향 벡터를 나타내고, $\boldsymbol{e}_{\gamma}$ 는 $\boldsymbol{e}_{v}$ 에 수직한 단위 방향 벡터로서 Longitudinal flight path angle의 증가 방향이며, 마지막으로 $\boldsymbol{e}_{\psi}$ 는 오른손 법칙에 따른 Yaw angle 증가방향의 단위벡터이다. 고정좌표계 (Fixed Inertial Frame)에서 제어축 좌표계로의 좌 표 변환 행렬 $\mathrm{D}$ 는 다음과 같이 나타낼 수 있다.

$$
D=\left[\begin{array}{ccc}
\cos \psi \cos \gamma & \sin \psi \cos \gamma & -\sin \gamma \\
-\sin \psi & \cos \psi & 0 \\
\cos \psi \sin \gamma & \sin \psi \sin \gamma & \cos \gamma
\end{array}\right]
$$

또한 식(3) (5)를 변환행렬 $\mathrm{D}$ 를 이용하여 제어축 좌표계로 나타내면 다음과 같다.

$$
\begin{gathered}
\Delta d-\Delta d^{*}=M_{v} e_{v}+M_{\psi} e_{\psi}+M_{\gamma} e_{\gamma} \\
v=v e_{v}
\end{gathered}
$$

$$
\frac{d v}{d t}=\dot{v} e_{v}+v \dot{\psi} \cos \gamma e_{\psi}-\dot{v} \dot{e_{\gamma}}
$$

여기서 $\left(M_{v}, M_{\psi}, M_{\gamma}\right)^{T}=D\left(M_{x}, M_{y}, M_{z}\right)^{T}$ 이다.

Leader와 Follower 무인기들 모두 Flight path 와 Yaw angle의 차이가 작다고 가정하면 Follower에서 Leader로의 좌표축 변환행렬을 아 래와 같이 나타낼 수 있다.

$$
\delta D=\left[\begin{array}{ccc}
1 & \delta \psi & -\delta \gamma \\
-\delta \psi & 1 & 0 \\
\delta \gamma & 0 & 1
\end{array}\right]
$$

여기서 $\delta \gamma=\gamma^{j}-\gamma^{k}$ 는 Flight path angle의 차이고, $\delta \psi=\psi^{j}-\psi^{k}$ 는 Yaw angle 차이다. 이것을 사용하 면 Follower의 제어축 좌표계 $\left(\boldsymbol{e}_{v}^{k}, \boldsymbol{e}_{\psi}^{k}, \boldsymbol{e}_{\gamma}^{k}\right)$ 에서의 Leader의 속도 벡터 $v^{j}$ 를 다음과 같이 표현할 수 있다.

$$
v^{j}=v^{j} \boldsymbol{e}_{v}^{k}+v^{j} \delta \psi \boldsymbol{e}_{\psi}^{k}-v^{j} \delta \gamma \boldsymbol{e}_{\gamma}^{k}
$$

이와 유사하게 Follower의 제어축 좌표계에서 Leader의 가속도 벡터는 다음과 같다.

$$
\begin{aligned}
\frac{d v^{j}}{d t} & =\left(\dot{v}^{j}-\dot{\psi}^{j} v^{j} \delta \psi \cos \gamma^{j}-\dot{\gamma}^{j} \delta \gamma v^{j}\right) \boldsymbol{e}_{v}^{k} \\
& +\left(\dot{v}^{j} \delta \psi+\dot{\psi}^{j} v^{j} \cos \gamma^{j}\right) \boldsymbol{e}_{\psi}^{k} \\
& +\left(-\dot{v}^{j} \delta \gamma-\dot{\gamma}^{j} v^{j}\right) \boldsymbol{e}_{\gamma}^{k}
\end{aligned}
$$

편대 유지의 목적은 무인기들 사이의 기하학 적 관계를 유지하는 것으로써 식(2)에 정의된 양 한정 함수를 감소하도록 유도 법칙을 선정하고자 한다. 이를 위하여, 식(2)를 미분하고 Follower의 제어축 좌표계로 다시 쓰면 다음과 같다.

$$
\begin{aligned}
\frac{d V}{d t} & =\left(\frac{d v^{j}}{d t}-\frac{d v^{k}}{d t}\right) \cdot\left(\Delta d-\Delta d^{*}\right) t_{g o} \\
& =\left(\dot{v}^{j}-\dot{\psi}^{j} v^{j} \delta \psi \cos \gamma^{j}-\dot{\gamma}^{j} \delta \gamma v^{j}-\dot{v}^{k}\right) M_{v}^{k} t_{g o} \\
& +\left(\dot{v}^{j} \delta \psi+\dot{\psi}^{j} v^{j} \cos \gamma^{j}-v^{k} \dot{\psi}^{k} \cos \gamma^{k}\right) M_{\psi}^{k} t_{g o} \\
& +\left(-\dot{v}^{j} \delta \gamma-\dot{\gamma}^{j} v^{j}+\dot{\gamma}^{k} v^{k}\right) M_{\gamma}^{k} t_{g o}
\end{aligned}
$$

여기서 요구되는 편대 비행 기하 오차 $\Delta d^{*}$ 를 상 수라 가정하였고, $\left(M_{v}^{k}, M_{\psi}^{k}, M_{\gamma}^{k}\right)$ 는 $\left(\Delta d-\Delta d^{*}\right)$ 의 성분으로서 Leader 및 Follower 비행체 사이의 현재 편대 비행 기하 오차이며 Follower의 제어 축 좌표계에서 표현된다. 식(13)의 음한정 (Negative Definiteness)을 보장하기 위하여 다음 과 같은 관계식을 갖도록 한다.

$$
\begin{aligned}
\frac{d V}{d t} & =-2 N^{k} V \\
& =-N^{k}\left(M_{v}^{k}\right)^{2}-N^{k}\left(M_{\psi}^{k}\right)^{2}-N^{k}\left(M_{\gamma}^{k}\right)^{2}
\end{aligned}
$$


여기서 $N^{k}$ 는 양의 상수이다. 예를 들어 Follower 의 동적 특성이 다음과 같은 조건을 만족하도록 제어된다면

$$
\begin{aligned}
& \dot{v}^{k}=\left(\dot{v}^{j}-\dot{\psi}^{j} v^{j} \delta \psi \cos \gamma^{j}-\dot{\gamma}^{j} \delta \gamma v^{j}\right)+\frac{N^{k}}{t_{g o}} M_{v}^{k} \\
& \dot{\psi}^{k}=\frac{\left(\dot{v}^{j} \delta \psi+\dot{\psi}^{j} v^{j} \cos \gamma^{j}\right)}{v^{k} \cos \gamma^{k}}+\frac{N^{k}}{t_{g o}} \frac{1}{v^{k} \cos \gamma^{k}} M_{\psi}^{k} \\
& \dot{\gamma}^{k}=\frac{\left(\dot{v}^{j} \delta \gamma+\dot{\gamma}^{j} v^{j}\right)}{v^{k}}-\frac{N^{k}}{t_{g o}} \frac{1}{v^{k}} M_{\gamma}^{k}
\end{aligned}
$$

위 식(15)를 식(13)에 대입하면 식(14)의 조건을 만족함을 알 수 있다. 즉, Follower UAV k의 동 역학이 식(15)와 같이 정의된 Model을 추종하도 록 제어되어야 한다.

본 연구에서는 유도 법칙 도출 과정의 단순화 를 위하여 각 $\mathrm{UAV} \mathrm{k}$ 의 동역학 및 제어 시스템 이 속도, 비행 경로각, 비행 Heading 과 같은 3 개 의 채널로 구성 하였고 이 식들은 간단한 1 차 함 수로 표현된다고 가정하였으며 아래의 식과 같이 표현하였다.

$$
\begin{aligned}
& \dot{v}^{k}=\frac{1}{\tau_{v}^{k}}\left(v_{c}^{k}-v^{k}\right) \\
& \dot{\psi}^{k}=\frac{1}{\tau_{\psi}^{k}}\left(\psi_{c}^{k}-\psi^{k}\right) \\
& \dot{\gamma}^{k}=\frac{1}{\tau_{\gamma}^{k}}\left(\gamma_{c}^{k}-\gamma^{k}\right)
\end{aligned}
$$

여기서 $\left(\tau_{v}^{k}, \tau_{\psi}^{k}, \tau_{\gamma}^{k}\right)$ 는 각 제어 루프들의 시상수이 고, $\left(v_{c}^{k}, \psi_{c}^{k}, \gamma_{c}^{k}\right)$ 는 유도명령들이다. 즉, 유도 명령 $\left(v_{c}^{k}, \psi_{c}^{k}, \gamma_{c}^{k}\right)$ 의 선정에 있어서 식(16)으로 정의되는 $\mathrm{UAV} \mathrm{k}$ 의 동역학 및 제어 시스템이 식(15)로 규 정된 Model을 추종하도록 선정해야 한다. 식(15) 및 식(16)에서 유도 명령은 다음과 같다.

$$
\begin{aligned}
v_{c}^{k}= & v^{k}+\tau_{v}^{k}\left(\dot{v}^{j}-\dot{\psi}^{j} v^{j} \delta \psi \cos \gamma^{j}-\dot{\gamma}^{j} \delta \gamma v^{j}\right) \\
& +N^{k} \frac{\tau_{v}^{k}}{t_{g o}} M_{v}^{k} \\
\psi_{c}^{k}= & \psi^{k}+\frac{\tau_{\psi}^{k}\left(\dot{v}^{j} \delta \psi+\dot{\psi}^{j} v^{j} \cos \gamma^{j}\right)}{v^{k} \cos \gamma^{k}} \\
& +N^{k} \frac{\tau_{\psi}^{k}}{t_{g o}} \frac{1}{v^{k} \cos \gamma^{k}} M_{\psi}^{k} \\
\gamma_{c}^{k}= & \gamma^{k}+\frac{\tau_{\gamma}^{k}\left(\dot{v}^{j} \delta \gamma+\dot{\gamma}^{j} v^{j}\right)}{v^{k}} \\
& -N^{k} \frac{\tau_{\gamma}^{k}}{t_{g o}} \frac{1}{v^{k}} M_{\gamma}^{k}
\end{aligned}
$$

식(17)은 궁극적으로 Follower UAV k에 필요 한 유도 명령이며, 이 유도 명령의 계산을 위하 여 식(17)의 오른쪽 정보가 필요하다. 이러한 정 보는 자신의 현재 비행 상태, 편대 비행 기하 오 차는 물론 Leader UAV의 비행 상태 정보까지 포함한다. 예를 들어 Leader UAV의 가속도 정 보 $\left(\dot{v}^{j}, \dot{\psi}^{j}, \dot{\gamma}^{j}\right)$ 도 필요하다. 그러나 만약 Leader $\mathrm{UAV}$ 의 동역학 및 제어 시스템이 식(16)과 같이 근사화할 수 있다면 이는 Leader UAV의 비행 상태 $\left(v^{j}, \psi^{j}, \gamma^{j}\right)$ 와 유도 명령 $\left(v_{c}^{j}, \psi_{c}^{j}, \gamma_{c}^{j}\right)$ 으로 대체할 수 있다. 이것은 Leader UAV의 비행 상태와 비 행 명령들이 Follower UAV로 전달되어야 한다 는 것을 의미한다. 위와 같은 구조의 유도 체계 에 있어서 특정 Follower UAV는 그가 속한 Branch내 모든 Leader UAV들의 비행 상태 및 유도 명령 정보는 물론 모든 Leader들 사이의 편대 비행 기하 오차 정보까지 활용하여 유도 명 령을 계산하게 된다.

\section{III. 시뮬레이션 및 결과 고찰}

\section{1 비선형 모델}

본 논문에서 제시한 유도법칙은 간단한 1 차 지연 시스템을 기반으로 한 비행체 모델을 근 거하고 있으나 유도법칙의 성능 파악을 위한 비행체 모델은 비선형 고정밀도 $6 \mathrm{DOF}$ 모델을 사용하였다. 본 논문에서는 참고문헌 [20]과 [21]에 제시된 비행체와 제어체계 모델을 사용 하였다

공력, 공력 모멘트, 그리고 추력은 비행 상태 및 비행 제어면 변위의 복잡한 함수이고 비선형 시뮬레이션에 모두 반영하였다. 제어면의 서보 모터는 1차 지연 시스템으로 모델링하였으며 Engine Dynamics와 Throttle Opening 또한 1차 지연 시스템으로 간략화 하였다.

비행 제어를 위한 각종 SAS 및 CAS는 그림 3 과 같이 설계하여 전체적으로 Speed, Flight path angle, Heading angle로 이루어진 3개의 제 어 채널만으로 비행 제어 시스템을 구성하였다.

그림 3 과 같이 표현된 $6 \mathrm{DOF}$ 비선형 비행체 동역학 및 제어 시스템은 System Identification 을 통하여 간단한 1차 지연 시스템으로 Model Matching할 수 있고 이를 통해 유도법칙에 필요 한 각 제어 채널의 시상수를 구하였다. 


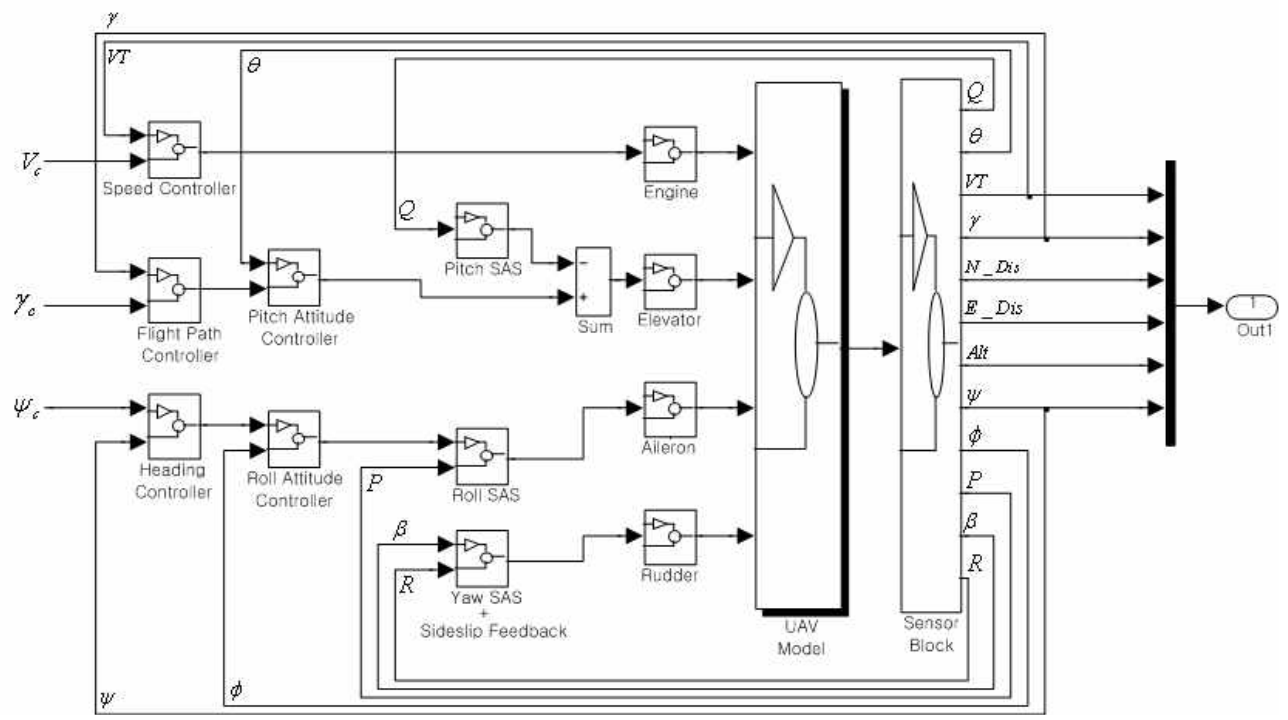

그림 3. UAV dynamics and Control System

\section{2 시뮬레이션 구성}

본 논문에서 제안한 유도 법칙의 성능 검증을 위하여 그림 4 에 보인 바와 같이 여섯 대의 $\mathrm{UAV}$ 들이 편대를 이루어 그림 5 와 같이 미리 지 정된 지역을 비행하는 정찰 및 감시 시나리오를 이용하였다. 특히 편대 전체의 Leader UAV는 표 1 에 보인 바와 같이 미리 설정된 비행 명령을 수행한다. 나머지 $\mathrm{UAV}$ 들은 그림 4에 설정된 바 와 같이 전후좌우 $\mathrm{UAV}$ 들에 대하여 일정한 편대 기하를 유지해야 한다.

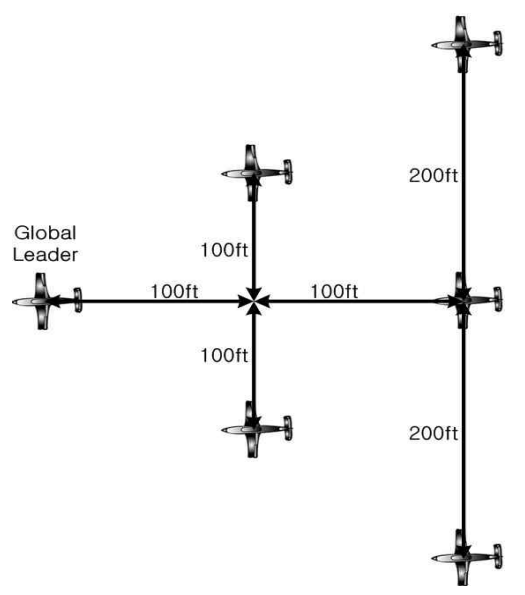

그림 4. Six(6) UAVs Formation Flying
Table 1. Flight Command Schedule for Reconnaissance and Surveillance Mission (Applied to the formation leader only)

\begin{tabular}{|l|c|c|l|l|l|}
\hline $\begin{array}{c}\text { Way } \\
\text { Point }\end{array}$ & Mission & $\begin{array}{c}\text { Time } \\
(\mathrm{sec})\end{array}$ & \multicolumn{1}{|c|}{$\begin{array}{c}\text { Speed } \\
(\mathrm{ft} / \mathrm{s})\end{array}$} & $\begin{array}{c}\text { Flight Path } \\
\text { Angle }\end{array}$ & \multicolumn{1}{|c|}{ Heading Angle } \\
\hline Wp.1 & $\begin{array}{c}\text { Target } \\
\text { Acquisition }\end{array}$ & $50\left(=\mathrm{t}_{1}\right)$ & $V_{c}=350$ & $\gamma_{c}=0^{\circ}$ & $\psi_{c}=0^{\circ}$ \\
\hline Wp.2 & Ingress & $350\left(=\mathrm{t}_{2}\right)$ & $V_{c}=350-\frac{1}{3}\left(t-t_{1}\right)$ & $\gamma_{c}=0.995^{\circ}$ & $\psi_{c}=0^{\circ}$ \\
\hline Wp.3 & Mission & $615\left(=\mathrm{t}_{3}\right)$ & $V_{c}=250$ & $\gamma_{c}=0^{\circ}$ & $\psi_{c}=\frac{495^{\circ}}{265}\left(t-t_{2}\right)$ \\
\hline Wp.4 & Ingress & $915\left(=\mathrm{t}_{4}\right)$ & $V_{c}=250+\frac{1}{6}\left(t-t_{2}\right)$ & $\gamma_{c}=-0.5209^{\circ}$ & $\psi_{c}=495^{\circ}$ \\
\hline Wp.5 & Mission & $1235\left(=\mathrm{t}_{5}\right)$ & $V_{c}=300$ & $\gamma_{c}=0^{\circ}$ & $\psi_{c}=495^{\circ}+\frac{450^{\circ}}{320}\left(t-t_{2}\right)$ \\
\hline Wp.6 & Disgress & $1350\left(=\mathrm{t}_{6}\right)$ & $V_{c}=300$ & $\gamma_{c}=0^{\circ}$ & $\psi_{c}=945^{\circ}$ \\
\hline Wp.7 & Return & 1600 & $V_{c}=300-\frac{1}{5}\left(t-t_{6}\right)$ & $\gamma_{c}=-0.625^{\circ}$ & $\psi_{c}=945^{\circ}$ \\
\hline
\end{tabular}

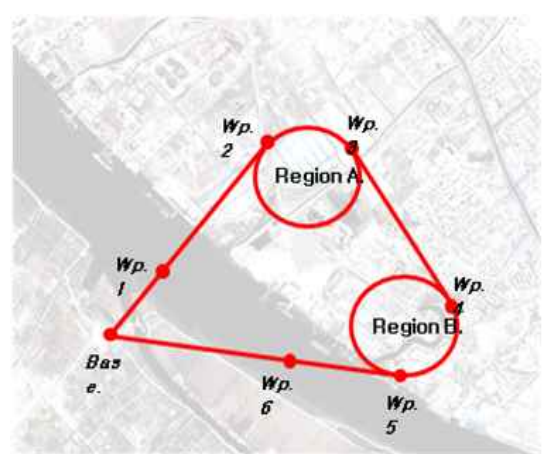

그림 5. Flight Trajectory for Reconnaissance and Surveillance Mission (plan view) 


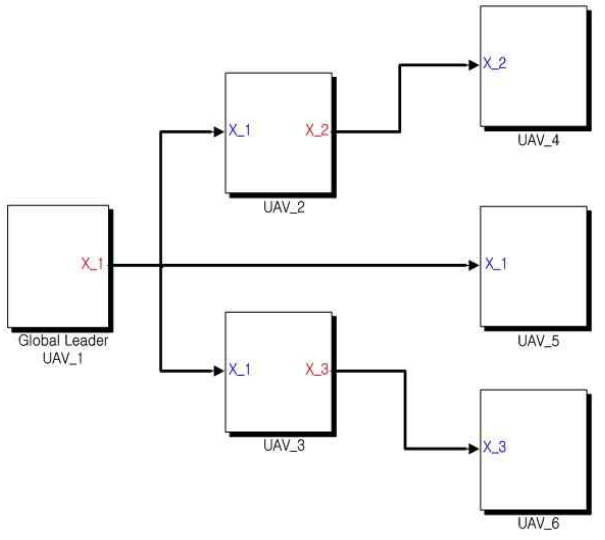

(a) Three branches

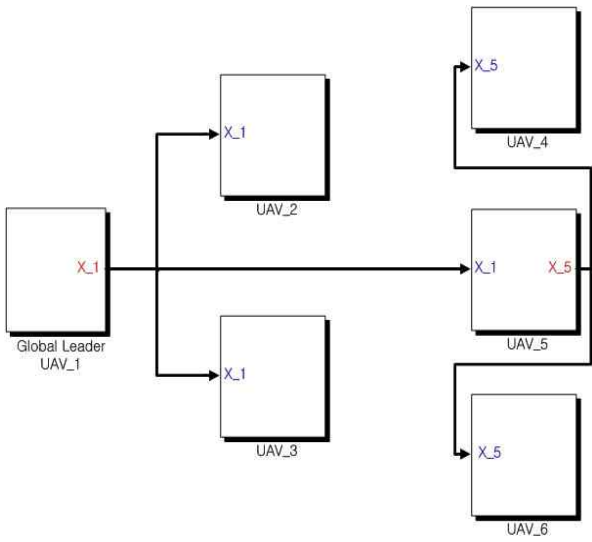

(b) Four branches

그림 6. Different Formation Architecture

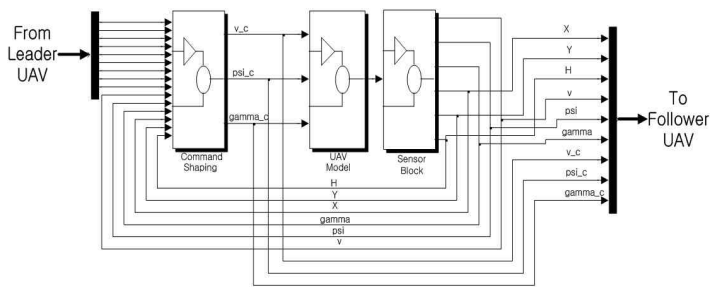

그림 7. Internal Structure of an UAV

각각의 UAV들이 어떻게 Local Leader와 Follower의 역할을 맡느냐에 따라 편대 구조를 결정할 수 있다. 그림 6 은 동일한 편대 기하에 대하여 Branch의 구성에 따라 두 가지 편대 구 성이 가능함을 예시하고 있다. 즉 (a)의 경우 3 개 의 Branch, (b)의 경우 4 개의 Branch로 구성되어 있다. 예를 들어, UAV 5는 Branch의 구성에 따
라 Follower의 역할만 또는 Leader의 역할도 동시 에 수행할 수 있다. 그림 6에서 각 블록은 한 대 의 $\mathrm{UAV}$ 를 나타내고 그림 7 은 내부 구조를 나타 낸다. 그림 7에서 "Command Shaping"은 식(17)을 사용하여 유도 명령을 계산하는 블럭이고 "UAV Model"은 그림 3에 제시한 Fully, Nonlinear 비행 체 동역학 및 제어 시스템을 의미한다.

\section{3 시뮬레이션 결과}

각각의 $\mathrm{UAV}$ 들은 모두 동일한 비행체이고 초 기에 모두 동일한 비행 상태를 유지하면서 그림 4에 보인 편대 기하를 유지하고 있다고 가정하였 다. 그림 8에서 여섯 대의 UAV들이 기동비행 중 에도 최초의 편대 기하를 유지하는 것을 알 수 있다. 그림 9는 각 비행체간의 시간변화에 따른 거리를 보이고 있다. 선회 구간을 제외하고 대부 분의 비행 구간에서 거의 일정한 거리를 유지함 을 확인할 수 있다. 그림 10 에서 12 까지는

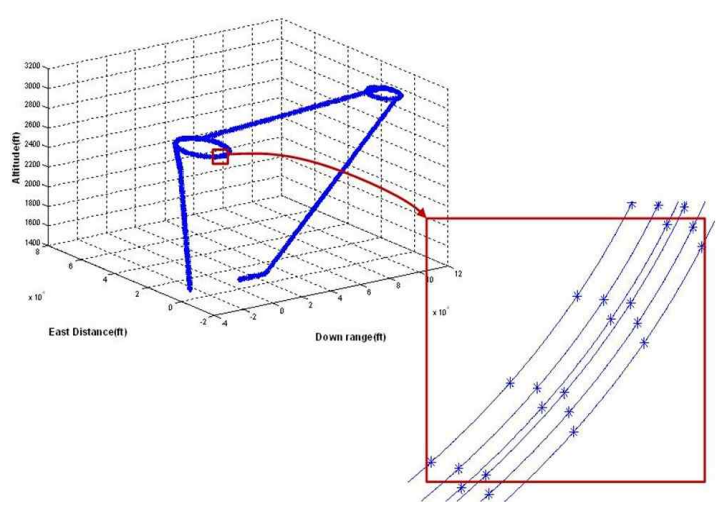

그림 8. Achieved Flight Trajectories

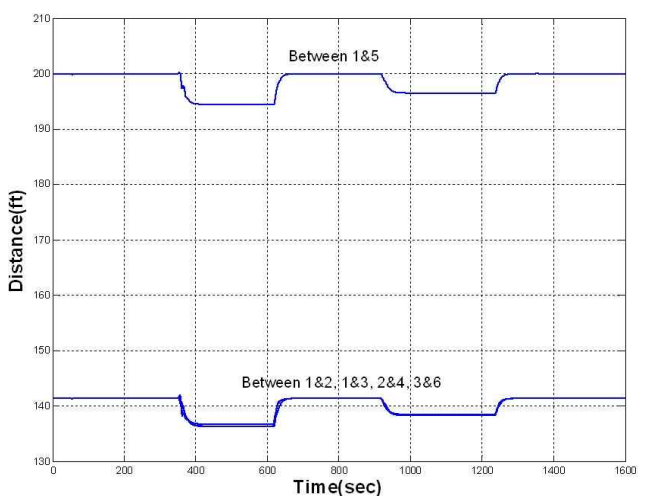

그림 9. Distance Between UAVs 


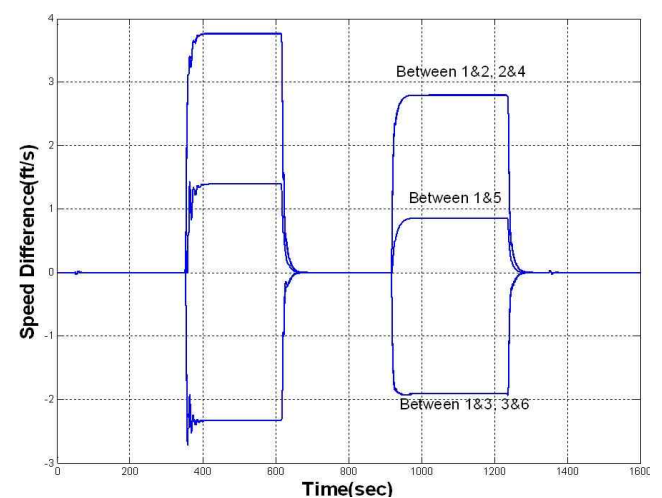

그림 10. Speed Difference Between UAVs

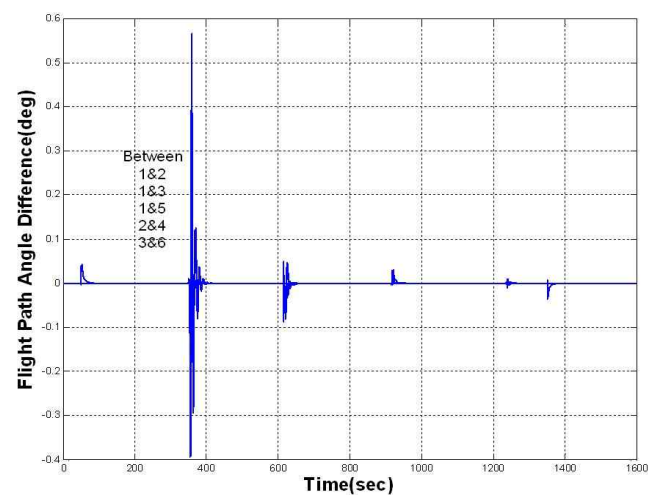

그림 11. Flight Path Angle Difference Between UAVs

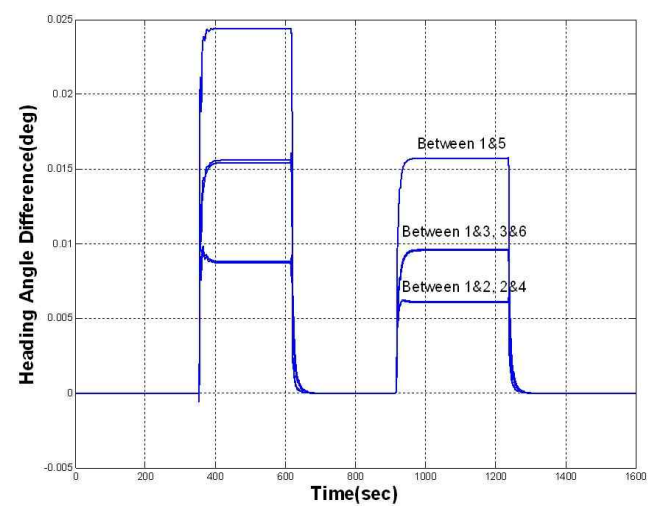

그림 12. Heading Angle Difference Between UAVs

Leader와 Follower UAV들 사이의 Flight speed, Flight path angle, Yaw angle 차이를 도시하고 있으며, 그 차이가 매우 작게 유지되고 있음을
알 수 있다. 이는 각 비행체들이 거의 동시 기동 을 하는 소위 Synchronized Flight Maneuver임 을 의미한다. 그러나 선회 비행 구간에서는 속도 및 Heading 각도가 일정한 오차를 보이고 있다. 이는 편대 기하를 유지하기 위하여 Global Leader에 대한 상대 위치에 따라 더 빠르게 혹 은 더 느리게 비행해야 하기 때문이다.

그림 6(a)과 6(b)이 거의 동일한 시뮬레이션결 과를 도출하여 본 논문에서는 그림 $6(\mathrm{a})$ 의 구조 에 대한 결과만을 제시하였다.

\section{$\mathrm{IV}$. 결 론}

본 논문에서는 다비행체(UAV) 편대 비행의 유도법칙을 제안하였고 비선형 고정밀도 시뮬레 이션을 통하여 제안된 유도법칙의 성능을 파악하 였다. 편대는 여러 Branch들로 이루어져있고 이 Branch에 속하는 개별 비행체는 Local Leader와 Follower의 역할을 갖는다. Follower 비행체는 자신의 비행 상태와 편대기하 오차뿐만 아니라 모든 Leader들의 비행 상태와 명령을 이용하여 자신에게 필요한 유도명령을 계산하는 Cascade 형식을 취하고 있다.

여섯 대의 $\mathrm{UAV}$ 들로 구성된 편대의 감시 및 정찰 시나리오에 대한 시뮬레이션을 수행하였으 며 결과적으로 편대내 모든 비행체가 동시 기동 비행(Synchronized Flight Maneuver)을 수행하여 효과적으로 전체 편대 기하를 유지하고 있음을 확인하였다.

\section{후 기}

본 연구는 방위사업청과 국방과학연구소의 지 원으로 수행되었으며 이에 대해 깊이 감사를 드 립니다. (계약번호 UD070041AD)

\section{참고문헌}

1) Development and Operation of UAVs for Military and Civil Applications, North Atlantic Treaty Organization, RTO Education Notes 9, April 2000.

2) Flint, M., Polycarpou, M., and Frenandez -Gaucherand, E., "Cooperative Control for Multiple Autonomous UAVs Searching for Targets", Proceedings of the 41st IEEE Conference on Decision and control, Las Vegas, 
Nevada, December 2002

3) Sarris, Z., "Survey of UAV Applications in Civil Markets (June 2001)", available on-line

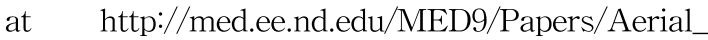
vehicles/med01-164.pdf

4) Bayraktar, S., Fainnekos, G. E., and Pappas, G. J., "Experimental Cooperative Control of Fixed-Wing Unmanned Aerial Vehicles", Proceedings of the 43rd IEEE Conference on Decision and Control, Atlantis, Paradise Island, Bahamas, December 2004.

5) How, J., King, E., and Kuwata, Y., "Flight Demonstrations of Cooperative Control for UAV Teams", AIAA 3rd "Unmanned Unlimited"Technical Conference, Workshop and Exhibit, Chicago, Illinois, 20-23 September 2004

6) King, E., Kuwata, Y., Bertuccelli, L., and How, J., "Coordination and Control Experiments on Multi-vehicle Testbed", Proceedings of the 2004 American Control Conference, Boston, Massachusetts, June 30 - July 3, 2004.

7) Campa, G., Seanor, B., Gu, Y., and Napolitano, M. R., "NLDI Guidance Laws for Close Formation Flight", 2005 American Control Conference, Portland OR, June 8-10, 2005.

8) Chandler, P. R., and et al, "Complexity in UAV Cooperative Control", Proceedings of the American Control Conference, Anchorage, AK May 8-10, 2002.

9) Rabbath, C. A., Gagnon, E., and Lauzon, M., "On the Cooperative Control of Multiple Unmanned Aerial Vehicles", IEEE Canadian Review, Spring 2004, pp. 15-19.

10) Bowen, D. G. and MacKenzie, S. C., "Autonomous Collaborative Unmanned Vehicles: Technological Drivers and Constraints", Defence R\&D Canada, Contract Report DRDC CR-2003 -003, September 2003

11) Verma, A., Wu, C.-N., and Castelli, V., "Autonomous Command and Control System for UAV Formation", AIAA Atmospheric Flight Mechanics Conference and Exhibit, Austin, Texas, 1-14 August 2003.

12) Bayraktar, S., Fainekos, G. E., and Pappas, G. J., "Hybrid Modeling and Experimental Cooperative Control of Multiple Unmanned Aerial Vehicles", Technical Report
MS-CIS-04-32, Department of Computer and Information Science, University of Pennsylvania, December 10, 2004.

13) Vachtsevanos, G., Tang, L., and Reimann, J., "An Intelligent Approach to Coordinated Control of Multiple Unmanned Aerial Vehicles", Presented at the American Helicopter Society 60th Annual Forum, Baltimore, MD, June 7-10, 2004.

14) Narayan, P., Wu, P., Campbell, D., and Walker, R., "An Intelligent Control Architecture for Unmanned Aerial Systems (UAV) in the National Airspace System (NAS)", 2nd Australian Unmanned Air Vehicle Systems Conference, March 20-21, 2007.

15) Sattigeri, R. and Calise, A. J., "An Adaptive Vision-Based Approach to Decentralized Formation Control", AIAA 2004-5252, AIAA Guidance, Navigation, and Control Conference and Exhibit, Providence, Rhode Island, 16-19 August 2004.

16) Campa, G., Napolitano, M. R., Seanor, B., and Perhinschi, M. G., "Design of Control Laws for Maneuvered Formation Flight", Proceedings of the 2004 American Control Conference, Boston, Massachusetts, June 30-July 2, 2004

17) Wan, S., Campa, G., Napolitano, M. R., Seanor, B., and Gu, Y., "Design of Formation Control Laws for Research Aircraft Models", AIAA 2003-5730, AIAA Guidance, Navigation, and Control Conference and Exhibit, Austin, Texas, 11-14 August 2003.

18) Segal, S., Ben-Asher, J. Z., and Weiss, H., "Derivation of Formation-Flight Guidance Laws for Unmanned Air Vehicles", Journal of Guidance, Control, and Dynamics, Vol. 28, No. 4, July-August 2005

19) Tahk, M.-J., Park, C.-S., and Ryoo, C.-K., "Line-of-Sight Guidance Laws for Formation Flight", Journal of Guidance, Control, and Dynamics, Vol. 28, No. 4, July-August 2005.

20) No, T. S., Min, B. M., Stone, R. H., and Wang, K. C., "Control and simulation of arbitrary flight trajectory-tracking", Control Engineering Practice, Vo. 13, 2005, pp. 601-612.

21) Stevens, B. L. and Lewis, F. L., Aircraft Control and simulation, John Wiley \& sons, Inc., U.S.A. 